\title{
How to Effectively Validate an HL7 Syndromic Surveillance Interface
}

\author{
Jeffrey Johnson*, Brit Colanter and Marjorie Richardson \\ Public Health Services, County of San Diego, San Diego, CA, USA
}

\section{Objective}

This presentation highlights the necessary steps to effectively validate a Health Level 7 (HL7) syndromic surveillance interface during the onboarding and implementation process.

\section{Introduction}

Current local, state, and national initiatives related to meaningful use and the modernization of electronic health records, and the growing availability of electronic information exchanges, have become important drivers to establishing syndromic surveillance systems. Effective implementation of electronic syndromic surveillance interfaces requires approaches that ensure the receipt of quality, timely, and reliable information.

While there are published specifications for the HL7 ADT message and National Institute of Standards and Technology (NIST) validation tools, there has been little documentation about the necessary steps for a local public health department to validate and confirm that an interface that is producing consistent and quality information. The lack of effective validation efforts has led to incomplete or inconsistent data utilized by syndromic systems and their intended audiences.

The County of San Diego has developed and utilized a framework for validating new syndromic interfaces. This presentation will highlight several pragmatic methods to validate the HL7 message content, provide specific examples of validation, and describe the pitfalls that could result from a poorly validated syndromic interface.

\section{Methods}

Message content validation involves reviewing, configuring and achieving meaningful content within the HL7 syndromic surveillance messages. The content validation process is viewed across similarly linked messages tied to patient encounter workflows.

The method of validation involves several validation domains that are applied to the syndromic messages on a per hospital basis. These validation methods are:

- Higher complexity syndromic use case development,

- Validation of selective priority constraining and encoding,

- Message timeliness and time sequence validation,

- Validity of trace back data elements,

- Completeness and validity of priority message segments,

- Validation of organization location information,

- Validation of content useful for syndromic category mapping,

- Internal consistency of the message, and

- External parallel validation with hospital medical records or other external surveillance systems.

When applied, these methods are often the catalyst for message reconfigurations. In turn, additional rounds of validation can be applied to ensure message changes meet criteria.

A set of syndromic message content validation guidelines have been developed to guide the validation work. These tools describe the corresponding steps to validation with criteria established to determine success or indicate improvements needed. In addition, a message content worksheet has been developed as a tool to track and document each data provider's validation activity and outcomes.

\section{Results}

During onboarding for syndromic surveillance, the County of San Diego actively engaged with hospitals, their electronic health record (EHR) vendors, and a local health information exchange (HIE). Numerous best practices have been identified. Hospitals have different information workflows, usage of message types, and assignments of patient status. When assigning a patient treatment location, several different approaches are used across hospitals or by the EHR rules-based workflows. These differences also include differing classifications of inpatient and ambulatory related messages, message segment values, and the sequence of timeliness regarding the patient's syndromic message versus the patient's actual encounter experience.

\section{Conclusions}

Public health agencies across the United States vary in their capacity to onboard meaningful use syndromic data. Public health agencies should not rely on EHR vendors alone or other system implementation surrogates to validate the quality of the syndromic information.

The County of San Diego has highlighted the importance and value in systematic validation of an HL7-based syndromic data source. Following these steps has led to optimized technical approaches to onboarding future hospitals, as well as insights into how the data can be used in a meaningful way. Preliminary outcomes have shown that it is valuable to have public health involved in the onboarding process and, more importantly, during the HL7 message content validation activities.

\section{Keywords}

Syndromic; surveillance; validation; interface; meaningful use

\section{*Jeffrey Johnson}

E-mail: Jeffrey.Johnson@sdcounty.ca.gov 